\section{Research methods}

Series editors J M Stephenson, A Babiker

\title{
Cohort studies in sexual health
}

\author{
Caroline A Sabin, Andrew N Phillips
}

\section{Introduction}

Examples of cohort studies in the sexual health literature are now increasingly common. The purpose of this article is to provide an overview of the design and analysis of cohort studies, along with a discussion of some of the potential problems with these studies which may be particularly relevant for cohorts which study factors related to sexual health. A comprehensive guide to the design and analysis of cohort studies can be found in the book by Breslow and Day. ${ }^{1}$

\section{What is a cohort study?}

A cohort study is a type of study in which a group of individuals is followed through time. Although cohort studies are frequently referred to in the literature, one of the distinguishing features of an epidemiological cohort study (as opposed to the literal meaning of the word "cohort" as a "group or gathering" of individuals), is that it is longitudinal - that is, there is some follow up of the individuals recruited to the study. Thus, many so called cohort studies are simply cross sectional studies, where a group of individuals is recruited for a one-off assessment, and are not followed any further. We will not consider these studies in this article.

The usual aim of a cohort study is to assess the relation between a specific event or disease outcome and exposure to one or more potential "risk" factors. The information on whether each individual has been exposed to these risk factors is usually collected at entry to the cohort, and the individuals recruited to the study are then monitored over time to see whether or not the event occurs. We may be interested in either the incidence of the event (that is, the rate of new events over a given time period), the average time until the event occurs, or the cumulative probability of developing the event at any time after recruitment to the cohort.

Broadly speaking, cohorts can be divided into two types. In the more traditional type of cohort study the event of interest is usually the development of a disease in individuals who were disease free at entry to the cohort. In this type of cohort we are often interested in the identification of factors which increase or decrease the risk of developing the disease. For example, Giuliani and colleagues ${ }^{2}$ carried out a study of the transmission of hepatitis $\mathrm{C}$ virus (HCV) in patients attending a clinic for HIV testing. In this study, the individuals included in the cohort were all HCV negative at entry and the event of interest was HCV seroconversion over follow up. The authors were interested in whether this was related to HIV status. A total of 709 individuals were included in the cohort and were tested on more than one occasion. In this group, $15 \mathrm{HCV}$ seroconversions occurred over a total follow up period of 886 person years, giving an incidence rate of 1.69 seroconversions per 100 person years of follow up. This rate was higher in those who were HIV positive than in those who were HIV negative.

The second type of cohort study is primarily concerned with individuals who already have a specific disease. In this type of cohort, researchers may wish to describe the natural history of disease in these individuals, consider changes in the course of disease over time, or describe responses to therapy in a real life clinical setting. Thus, the event of interest in this type of study will not be the incidence of disease itself (individuals already have the disease of interest) but will reflect other disease outcomes such as progression of disease, death, or response to therapy. For example, Augenbraun $^{3}$ followed a cohort of 525 patients with early syphilis at yearly intervals to assess differences in the apparent response to treatment measured by two different assays, the microhaemagglutination assay for antibodies to Treponema pallidum and the fluorescent treponemal antibody absorption test. All patients recruited to the study had the disease (syphilis) at entry and the primary outcome of interest in this study was seroreversion, as measured by each of the assays, which would indicate that treatment had been effective.

In general, design issues for these two different types of cohort studies are similar, although they may differ in terms of the types of patients recruited to the cohort. Throughout the remainder of this article, therefore, we will stick to a single terminology and will refer to "outcome" to mean either the onset of disease, or the event of interest.

\section{Why perform cohort studies?}

Although cross sectional studies are quick and relatively cheap to perform, they suffer from two main limitations. Firstly, unless the study is repeated on the same individuals at some future time point, they cannot be used to assess the incidence of an outcome, although the prevalence of the outcome can be estimated. Secondly, although relations between a potential risk factor and the outcome can be investigated, the temporal relation between the two cannot be assessed - that is, it is not possible to tell whether exposure to the risk factor 
occurred before or after the outcome. Thus, any inferences about the causal role of the risk factor are limited. Because of the longitudinal nature of a cohort study, it is possible to assess the incidence of the outcome in these individuals over a given time period, and the temporal relations can be clarified.

\section{What are the disadvantages of cohort studies?}

Unfortunately, the benefits of a cohort study must come at a cost, and this is usually in terms of the size of the study and length of the study period required. When an outcome is relatively rare, cohorts often have to be very large and individuals must be followed for many years before sufficient numbers have experienced the outcome to enable an analysis of the impact of the risk factor to be performed. This is often the case for epidemiological studies of the incidence of diseases such as cancer and heart disease in the general population where cohorts tend to follow many thousands of individuals for periods in excess of $10-20$ years. $^{45}$ This long time period has major implications for the running of a cohort study: the longer the period of follow up, the greater the chance that members of the cohort will leave the study, either because they die from other causes before the event has occurred or because they choose to leave the study for other reasons, and it becomes harder to maintain consistency in the measurement of both the risk factor and outcome over time (see below). Therefore, if the outcome is particularly rare then other study designs, such as case-control studies, ${ }^{6}$ may be more appropriate. Alternatively, it may sometimes be possible to create a cohort retrospectively (see below).

\section{Design issues for cohort studies of sexual health}

As with any other study, cohorts should be precisely defined at the outset of the study. Thus, care should be taken to specify the nature of the individuals to be recruited to the cohort, the time point at which they enter the cohort (for example, a certain calendar date or, in those with disease, the date of diagnosis or date of presentation), whether the cohort is fixed (that is, new cohort members are not recruited as other members leave the cohort) or dynamic/ open (that is, new members continue to be recruited to the cohort throughout follow up), and how frequently the cohort is to be monitored.

WHICH PATIENTS SHOULD BE RECRUITED? It is of particular importance to specify the population which is to be studied. Individuals recruited to the cohort study should be broadly representative of this population (although this may often be more difficult than it sounds as individuals who agree to take part in cohort studies may differ from those who choose not to take part). When the primary aim of a cohort is to assess the incidence of disease and its relation to risk factors, then the population of interest may be the general population (although this isn't always the case). For example, the Dunedin Multidisciplinary Health and Development Study is a cohort study of the health, development, and behaviour of a cohort of young people from New Zealand, who were recruited at the age of 3 years from the general population. Over a number of years, the participants have been interviewed about factors relating to sexual health, including sexual behaviour, timing of first intercourse, ${ }^{7}$ and number of sexual partners. ${ }^{8}$ As this cohort is broadly representative of the general population in New Zealand, the results can be generalised to other young individuals born at the same time in the country. However, there are a number of problems which are encountered when studying sexual health in the general population. The intimate nature of sexual health means that recruiting individuals to cohort studies and maintaining follow up on these individuals can often be hard and, as a result, the cohort may be less representative of the general population than desired. In addition, the risk of sexually transmitted infections may be quite low in many individuals in the general population, meaning that such cohorts will have to be relatively large. For these reasons, researchers have often chosen to study alternative populations in whom these issues may be of less concern. For example, cohort studies have often focused on individuals who are either at high risk of sexually transmitted diseases (STDs), such as attenders at STD clinics ${ }^{10}$ or sex workers, ${ }^{11}$ or those who can be followed up easily, such as military conscripts ${ }^{12}$ or employees of the same company. ${ }^{13}$ The former group, in particular, has the additional advantage of being more used to discussing sexual health matters, and therefore cohort studies are often easier to conduct in this setting. While these cohorts are often less representative of the general population, and the results may not always be generalisable to the general population, the benefits of selecting these alternative populations usually outweigh the need for a truly general population sample. However, the individuals recruited to these studies should still be representative of the populations from which they are drawn.

It is not only the representativeness of the patients which should be considered. For example, the HIV epidemic first started in the United Kingdom from 1979 onwards, although an HIV test did not become available until 1985. When this test did become available many individuals were diagnosed with HIV and were recruited to cohort studies. At this time, therefore, many of the individuals recruited to these cohorts may have been infected with HIV for up to 6 years. The concern with cohorts such as this is that any individuals who died before the introduction of the HIV test would not have been tested and would be unlikely to be recruited to the study (unless HIV status could be ascertained from some other source). Thus, unless the cohort investigators pay particular attention to ensuring that all infected individuals were included in the study (that is, by establishing the HIV status of all patients who died before 1985), these cohorts may be biased towards "slower progressors"-that is, 
individuals who remained alive for at periods of up to 6 years after seroconversion. A fuller description of this type of survivorship bias is provided by Porter et al. ${ }^{14}$

RETROSPECTIVE COHORTS

Although cohorts are usually prospective (that is, they follow individuals forward in time), cohorts may also be retrospectively identified, often through a review of patient records or from other sources. For example, Blank and colleagues ${ }^{15}$ constructed a retrospective cohort of 3579 women jailed multiple times in New York City. Information was collected from syphilis testing records held at the prison. The benefits of such cohorts is that they are usually relatively cheap to create and the outcome may already be known for many individuals at the time the cohort has been formed. Thus, the time period required is far shorter. However, the main worry with retrospective cohorts such as this is that information recorded in patient records may not always be of high quality, and thus some details may be lacking. In addition, in some cases, the fact that the outcome is already known for many patients may bias the amount and type of information that is collected.

\section{The analysis of cohort studies for the impact of a risk factor on outcome}

The simplest approach to the analysis of a cohort study involves the calculation of the relative risk ( $\mathrm{RR}$ ), which measures the increased (or decreased) risk of the outcome in the exposed group compared with the unexposed group.

The risk of the outcome is estimated as the number of individuals in the cohort who have the outcome over the study period, divided by the total number of individuals in the cohort. This risk can be estimated separately for those who are exposed to the factor of interest $\left(\right.$ risk $\left._{\text {exp }}\right)$ and for those who are unexposed $\left(\right.$ risk $\left._{\text {unexp }}\right)$. The RR is then calculated as the ratio of the risks in the two groups ( risk $_{\text {exp }} /$ risk $_{\text {unexp }}$ ). The RR will be equal to one if the risk is the same in the two groups, greater than one if the risk is higher in the exposed than unexposed group, and less than one if the risk is lower in the exposed than unexposed groups. Confidence intervals can be calculated for the RR, and significance tests performed to see if the $\mathrm{RR}$ is significantly different from one.

There are a number of other methods of estimating the RR which may take into account the length of follow up in the exposed and unexposed groups. So, rather than expressing the risk as a percentage of patients under follow up, it may be expressed as a rate per 100 person years. The relative rate is calculated similarly to the $\mathrm{RR}$, by calculating the ratio of the rates in the two groups. In some situations it may actually be the time until an outcome occurs which is of primary interest, rather than simply whether or not the outcome occurred (for example, in studies of the natural history of disease, the question of interest may be whether the time to disease progression is more rapid in some groups of patients than in others). If this is the case then survival analysis methods may be used to obtain the cumulative probability of having the outcome at any point in time, and therefore the median time until the outcome occurs (the time point by which the outcome has occurred in $50 \%$ of the patients). Plots of this probability over time, known as Kaplan-Meier, life table, or survival curves, can be compared in different groups using the log rank test. Proportional hazards regression methods can be used to estimate the relative hazard of the outcome in the two groups, which is interpreted in a similar manner to the RR. A full description of these survival methods is beyond the scope of this article, but further details can be found in Cox and Oakes. ${ }^{16}$

Statistical modelling methods means that it is also possible to adjust the relative rate for other potential cofactors. For example, a study by Wong and $\mathrm{Chan}^{17}$ considered the relation between the prevalence of pharyngeal gonorrhoea over a 6 month period and condom use for oral sex among female brothel based sex workers in Singapore. The study recruited 836 sex workers who were free of pharyngeal gonorrhoea at the start of the study. The women had monthly pharyngeal swabs taken for $N$ gonorrhoeae culture. Over a 6 month follow up period, 25 of the 213 women $(11.7 \%)$ who reported inconsistent condom use had developed pharyngeal gonorrhoea compared with only 13 of the 506 women (2.6\%) who reported consistent condom use. This gave an unadjusted RR of 4.6 (25/213 divided by 13/506). Thus, women who reported inconsistent condom use were almost five times as likely to develop pharyngeal gonorrhoea as those who reported always using a condom. However, women who report inconsistent condom use may differ from those who report consistent condom use, both in terms of their demographics (for example, age, ethnicity) and previous experience as a sex worker. Some of these factors may also be risk factors for pharyngeal gonorrhoea (for example, those who had worked previously as sex workers had a lower rate of pharyngeal gonorrhoea than those who had recently entered sex work for the first time). These factors are known as confounding factors in the analysis, as the apparent relation between inconsistent condom use and pharyngeal gonorrhoea could be "explained" by differences in these factors. After adjusting for these confounding factors the relative risk increased dramatically to 17.1 .

\section{Measurement of risk factors and outcomes}

Clearly, it is important to obtain accurate information on exposure to the risk factors of interest and on the outcome itself. If the information collected is not accurate then, at best, the relation between the risk factor and outcome will be weakened and, at worst, the results will be seriously biased. The longitudinal nature of cohort studies, however, means that this may be difficult for a number of reasons.

Cohort studies generally record information on risk factors at entry to the study and then 
relate these to an outcome, which may occur many months or years after entry to the study. So, for example, information on sexual behaviour and previous infection with viral pathogens may be collected at entry to a study. This information may then be related to an outcome that may occur up to 10 years later. As cohort members age, their sexual behaviour and their viral status are likely to be modified. The question being addressed in a study of this type, therefore, relates only to the long term relation of a single exposure measurement at one point in time and the outcome, and may underestimate the true role of these factors in the development of the outcome. Thus, where risk factors are likely to change, attempts should be made to collect information on these risk factors at regular time intervals over the study period, so that the most up to date information can be incorporated into any analysis.

The measurement of the risk factor itself may also be changing. For example, assays to detect previous infection with viral agents are constantly being improved, and generally become more sensitive and specific over time. Thus, information on infection status in individuals tested with first generation tests may differ from that measured using second or third generation tests. Unless there are stored samples available, which is relatively unusual, information collected at entry to the study may become increasingly unreliable over time.

It is not only exposure to the risk factor that may change over time, but the outcome itself. For example, in the 20 years of the AIDS epidemic, the definition of AIDS has changed on a number of occasions. Each time a change in definition occurs, the incidence of AIDS increases simply because the definition includes more individuals who would previously have been AIDS free. ${ }^{18}$

\section{Dropouts and loss to follow up}

Because of the length of time required to complete a cohort study, one of their major limitations is that individuals may drop out of the cohort at various stages for any number of reasons. If the individuals who drop out are a random sample of the whole cohort then this is unlikely to bias the results, although the size, and therefore the power, of the cohort will be reduced. However, there is a potential for bias if certain types of individuals are more likely to drop out than others. This will mean that the cohort may become less representative of the population as time progresses, and ultimately the benefit of the cohort design will be lost. In addition, if those at greatest risk of the outcome tend to be those who drop out, then the incidence of the outcome will be underestimated. If there is a further tendency for these people to be exposed (or unexposed) to the risk factor, then additional bias will occur. For example, in the study by Wong and $\mathrm{Chan}^{17}$ described above, $17.8 \%$ of the women were lost to follow up because they decided to quit prostitution and couldn't be followed over the entire 6 month period. A greater proportion of non-Chinese sex workers were lost to follow up $(38.3 \%)$ than Chinese sex workers $(11.9 \%)$. As those non-Chinese sex workers who remained under follow up were more likely to develop pharyngeal gonorrhoea than Chinese sex workers, the authors were concerned that the cumulative incidence of pharyngeal gonorrhoea at 6 months may have been underestimated.

\section{Making treatment comparisons in cohort studies}

It is often tempting to use cohort studies to consider the effect of treatment on disease progression. The most common way of doing this is to consider whether the incidence of disease has changed over time, in line with the introduction of novel therapies or other therapeutic advances. For example, Macaluso and colleagues $^{19}$ described the effect of a behavioural intervention promoting barrier methods, and free barrier contraceptives on the incidence of gonorrhea and chlamydia among women attending an STD clinic. Because barrier protection was used in more than $70 \%$ of reported acts of vaginal intercourse, the authors concluded that "the study protocol was successful in promoting consistent and proper use of barrier methods." However, no control group was included and thus it is difficult to conclude that it was the intervention that was solely responsible for the change in behaviour. Occasionally, non-treated patients are used as a pseudo-control group. However, patients who choose not to be treated in a cohort study are often very different from those who are treated, either in terms of their disease status or their demographic and lifestyle factors. Ideally, estimates of treatment effects should be made from randomised controlled trials. However, where treatment comparisons are to be made in observational cohort studies, they should be interpreted carefully. ${ }^{20}$

\section{Conclusions}

Despite their relatively high cost, cohort studies are the only means by which to study the incidence of an outcome over a given time period. In addition, cohort studies provide an extremely valuable resource for monitoring the longitudinal changes in incidence of an outcome, for studying the natural history of disease, and for assessing response rates to treatment in a real life clinical setting. However, the longitudinal nature of cohort studies means that, in order to maintain the value of the cohort design, care should always be taken to minimise drop outs, and to ensure consistency in the measurement of the risk factor and outcome over the period of study.

Both authors co-wrote the article.

1 Breslow NE, Day NE. Statistical methods in cancer research. Volume II-The design and analysis of cohort studies. Oxford: University Press/IARC, 1987.

2 Giuliani M, Caprilli F, Gentili G, et al. Incidence and determinants of hepatitis $\mathrm{C}$ virus among individuals at risk of sexual transmitted diseases attending a human immunodeficiency virus type 1 testing program. Sex Transm Dis 1997;24:533-7

3 Augenbraun M, Rolfs R, Johnson R, et al. Treponemal specific tests for the serodiagnosis of syphilis. Syphilis and HIV Study Group. Sex Transm Dis 1998;25:549-52.

$4 \mathrm{Hu}$ FB, Manson JE, Liu S, et al. Prospective study of adult Hu FB, Manson JE, Liu S, et al. Prospective study of adult
onset diabetes mellitus (type 2) and risk of colorectal cancer in women. $\mathcal{F}$ Natl Can Inst 1999;91:542-547. 
5 Kannel WB. Fifty years of Framingham Study contributions to understanding hypertension [review]. F Hum Hyperten

6 Breslow NE and Day NE. Statistical methods in cancer research. Volume I-The analysis of case-control studies. Lyons: IARC, 1980

7 Dickson N, Paul C, Herbison P, et al. First sexual intercourse: age, coercion and later regrets reported by a birth cohort. BMF 1998;316:29-33.

8 Dickson N, Paul C, Herbison P. Adolescents, sexual behaviour and implications for an epidemic of HIV/AIDS among the young. Genitourin Med 1993;69:133-40.

9 Prins M, Hooykaas C, Coutinho RA, et al. Incidence and risk factors for acquisition of sexually transmitted diseases in heterosexuals with multiple partners. Sex Transm Dis 1994;21:258-67.

10 Diamond C, Selke S, Ashley R, et al. Clinical course of patients with serologic evidence of recurrent genital herpes presenting with signs and symptoms of first episode presenting with signs and symptom

11 Ward H, Day S, Weber J. Risky business: health and safety in the sex industry over a 9 year period. Sex Transm Inf 1999 ; the sex ind $340-3$

12 Celentano DD, Nelson KE, Suprasert S, et al. Epidemiologic risk factors for incident sexually transmitted diseases in young Thai men. Sex Transm Dis 1996;23:198-205.
13 Rakwar J, Jackson D, Maclean I, et al. Antibody to Haemophilius ducreyi among trucking company workers in Thsm Dis 1997;24:267-71.

14 Porter K, Johnson AM, Phillips AN, et al. The practical significance of potential biases in estimates of the AIDS incubation period distribution in the UK Register of HIV Seroconverters. AIDS 1999;13:1943-51.

5 Blank S, Sternberg M, Neylans LL, et al. Incident syphilis among women with multiple admissions to jail in New York City. F Infect Dis 1999;180:1159-63.

16 Cox DR, Oakes D. Analysis of survival data. London: Chapman and Hall, 1984

17 Wong ML, Chan RKW. A prospective study of pharyngeal gonorrhoea and inconsistent condom use for oral sex among female brothel-based sex workers in Singapore. Int F STD AIDS 1999;10:595-9.

18 Pezzotti P, Napoli PA, Rezza G, et al. The effect of the 1993 European revision of the AIDS case definition in Italy: implications for modelling the HIV epidemic. AIDS 1997; 11:95-9.

19 Macaluso M, Artz L, Kelaghan J, et al. Prospective study of Macaluso $\mathrm{M}$, Artz L, Kelaghan J, et al. Prospective study of
barrier contraception for the prevention of sexually transmitted diseases: study design and general characteristics of the study group. Sex Transm Dis 1999;26:127-36.

20 Sabin CA, Phillips AN. Treatment estimates in observational databases. F Antimicrob Chemo 2001 (in press).

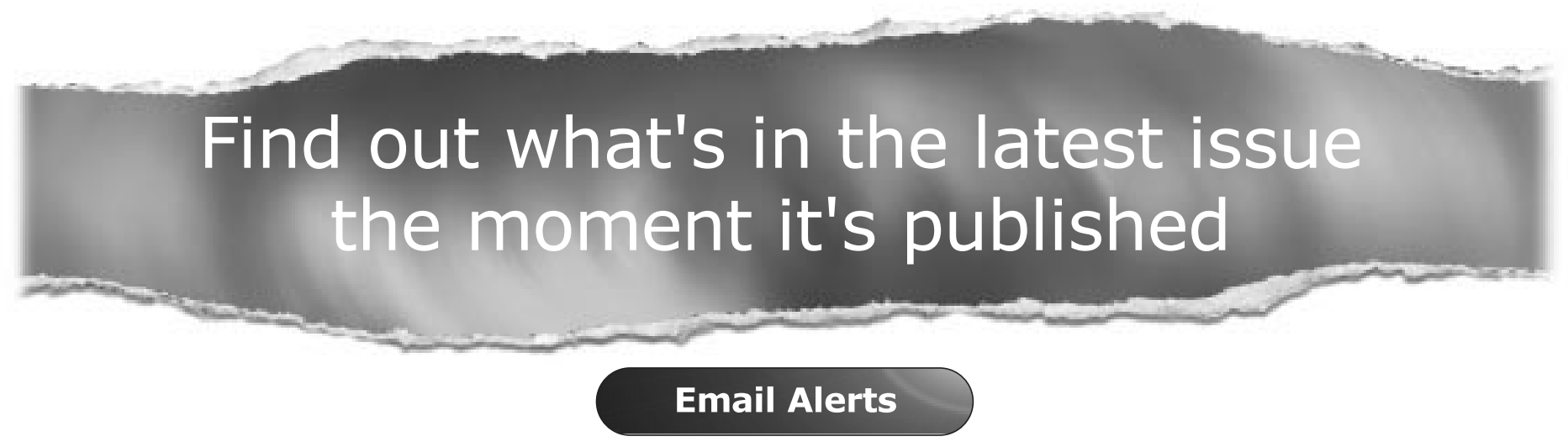

Sign up to receive the table of contents by email every month. You can select from three alerts: Table of Contents (full), TOC Awareness (notice only); Sexually Transmitted Infections related announcements.

www.sextransinf.com 